\title{
Comparaison des lois de Gumbel et de Fréchet sur l'estimation des débits maxima de crues
}

\author{
Comportement asymptotique des courbes de débits classés (1)
}

\section{Comparison between Gumbel's and Fréchet's laws on the estimation of maximum flood discharges}

\author{
The asymptotic behaviour of classified discharge curves
}

\author{
PAR M. BERNIER
}

INGÉNIEUR AU. SERYICE DES ĹTUDES ET RECHERCHES HYDRAHLIQUES D'ÉLECTHIGITÉ DE FRANCE

Tommunication présentée lors de la réunion partielle de la Société Hydrotechnique de France (Section d'Hydro107 ie statistique. - Commission pour l'étude des débits de crue) organisée à Paris le 12 septembre 1957.

\begin{abstract}
La theorie statistique des valeurs extremes a montré que deux types de lois de probabilité sont susceptibles d'être ajustées anx distributions de fréquence des débits de crue: ce sont les lois de Gumbel et de Fréchet. Il est en général très difficile de faire un choix cohérent entre ces deux lois a partir des trop conrtes séries d'observations de débits maxima annuels dont on dispose actuellement.

On étudie ici certaines propriétés asymptotiques des courbes de débits classés qui pourraient etre utilisés pour le choix a priori entre les lois de Gumbel et de Fréchet. Cetle citude comporte :

a) Des ajustements de lois de Galton composés aux courbes de débits classés observés de huit cours d'ean a régimes varies.

b) La comparaison de ces lois avec la distribution de Gumbel pour les grandes valeurs des débits.

En conclusion, le plus ou moins bon ajustement de la loi de Gumbel aux débits de crue, semble être lié à certaines caractéristiques hydrologiques des bassins étudiés. Cette liaison demandera cependant $\dot{a}$ etre précisée par des applications a des exemples plus nombreax et par un perfectionnement de la méthode statistique utilisée
\end{abstract}

The statistical theory of extreme values has shown that the Gumbel and Frehet probability laws can be made to fit flood discharge frequency distributions. It is usually very difficult to base a coherent decision as to which of these two laws to use on presently available maximum annual discharge data which does not cover sufficiently long periods.

Certain asymptotic properties of classified discharge curves, which can be used to make an a priori choice between the Gumbel and Frechet laws, are studied here. This study includes:

a) Examples of fitting compound Galton laus to classified discharge curves for eight streams having varying regimens.

b) A comparison of these laus with the Gumbel distribution for high discharge values.

In conclusion how well the Gumbel law can be made to fit flood discharges, seems to be dependent upon certain hydrological characteristics of the river basins being investigated. However, this connection will have to be determined by applying the procedure to a greater number of examples and perfecting the slatistical method which is used.
Dans la présente note, nous nous proposons ubludier un aspect particulier de la courbe des

(1) M. le Professeur" Gumber a traité le 23 avril 1950 Gevant la Société Hydrotechnique de France, la théorie Tos valeurs extrêmes appliquée aux débits maxima de trite. Cot exposé avait été suivi d'une discussion au cours 16 laquelle $M$. Bernier avait, dans une importante interNention, comparé la loi de Gumbel à diverses autres lois roulton-Gibrat, Fréchet) dans leurs applications à l'éva- débits classés : son comportement pour les gran. des valeurs du débit.

luation des débits maxima de crues. Ces exposés ont paru dans la Houille Blanche de 1956 , n* 5 .

An cours d'une seconde rénnion de la S.H.F., le 12 septembre 1957, M. Bernier présenta un nouvel exposé sur la comparaison des lois de Gumbel et de Fréchet, landis que M. Gumber consacrait aux débits d'étiage une communication qui constitue une application inverse de Ia théorie des valeurs extrêmes. (Cf. p. 57 du présent numéro.) 
Rappelons que les applications de la théorie des valeurs extrêmes nous ont montré (2) que deux types de lois de probabilité (lois de Gumbel et Fréchet) peuvent s'ajuster aux courbes de fréquences empiriques des débits de crue. Le problème du choix a priori entre ces deux lois se pose donc.

En effet, les formes de ces deux lois sont très voisines pour les valeurs centrales et ne different essentiellement que par leurs comportements asymptotiques. Il est par ce fait impossible de détecter des différences sur les trop courtes séries d'observations de crue existantes, qui ne renseignent précisément que sur les parties centrales des distributions.

Théoriquement, le choix entre les lois de Fréchet et Gumbel, dont les fonctions de répartition s'expriment simplement en fonction de variables réduites :

$$
\begin{aligned}
& \mathrm{G}(y)=\exp \left[-e^{-y}\right] \\
& \mathrm{H}(z)=\exp \left[-z^{-k}\right]
\end{aligned}
$$$$
\text { (k positif) }
$$

dépend de la forme analytique prise par la loi initiale des débits journaliers pour les grandes valeurs. On ne peut confondre cette loi, telle qu'elle est définie dans la théorie de Gumbel, avec l'expression mathématique de la courbe des débits classés, étant donné l'hétérogénéité de celle-ci résultant du mélange des débits d'années différentes et des liaisons entre débits de jours successifs. On conçoit cependant, de manière iniuitive, que le comportement de cette courbe pour les grandes valeurs du débit est identique a celui de la loi initiale des débits journaliers ou, mieux, à celui de la loi des crues annuelles.

Nous ferons donc cette hypothèse, qui peut d'ailleurs être vérifiée par certaines justifications théoriques.

Le comportement asymptotique de la courbe des débits classés guidera done le choix entre les deux lois des valeurs extrêmes.

L'étude de ce comportement est effectuée ici en plusieurs étapes:

a) Ajustement d'une loi de probabilité assez simple pour représenter convenablement les grandes valeurs, à la courbe observée des débits classés de certains cours d'eau. Critique de l'ajustement.

b) Etude des distributions des valeurs extrêmes d'échantillons tirés au hasard de la loi ainsi ajustée. Ajustement des lois de Gumbel et Fréchet à ces distributions dans la zone du champ de variation des débits maxima annuels.

\section{I. - REPRESENTATION MATHEMATIQUE DE LA COURBE DES DÉBITS CLASSES}

\section{1. - Choix d'un type de loi.}

Il y a intérêt à remplacer la courbe des débits classés observée, qui n'est pas d'un maniement facile, par une forme mathématique convenable. Cette loi de probabilité théorique doit être assez souple, de façon à représenter fidèlement les grands débits. L'utilisation d'une simple loi de Galton, comme l'a préconisé M. Gibrat, ne s'est pas avérée satisfaisante dans le cas présent. Il est préférable d'employer une loi mixte (composition de deux lois de Galton), tenant compte ainsi de l'hétérogénéité des éléments constitutifs de la courbe des débits classés. Cette loi mixte a d'ailleurs le mérite de posséder un plus grand nombre de paramètres, d'où sa plus grande souplesse.

(2) La Houille Blanche, $\mathrm{n}^{\circ}$ 5, 1956, page 718 .

\section{2. - Ajustement de la somme de deux lois de Galton.}

Les applications de cette loi, déjà étudiée par M. Bloch, n'ont pu être généralisées par suite des complications de la méthode de calcul des paramètres mise au point par cet auteur. Mais les calculs peuvent être de beaucoup simplifiés si on utilise comme donnée initiale le débit logarithmique (logarithme du débit suivant la dénomination d'Halphen) à la place du débit réel.

Soit donc $x$ le débit logarithmique.

On considère la fonction de répartition :

$$
\mathrm{F}(x)=1 / 2 \mathrm{~F}_{1}(x)+1 / 2 \mathrm{~F}_{2}(x)
$$

(on suppose, pour simplifier, même répartition de deux lois composantes, d'où le facteur $1 / 2$ ).

Dans cette formule :

$F_{1}$ est une loi normale (moyenne $\mu_{1}$, écart-type $\sigma_{1}$ ). 
$F_{2}$ est une loi normale (moyenne $\mu_{0}$, écart-type $\sigma_{2}$ ).

11 s'agit done d'estimer $\mu_{1}, \mu_{.2}, \sigma_{1}, \sigma_{2}$.

De la façon classique, on les détermine en fonction de la moyenne $m$, de la variance $\sigma^{2}$ et des coefficients de Pearson $\beta_{1}$ et $\beta_{2}$ de la distribution empirique des débits observés :

Il est commode de poser :

$$
\begin{gathered}
\lambda=\operatorname{tg} 0=\sigma_{1} / \sigma_{2} \quad\left(\sigma_{1} \geqslant \sigma_{2}\right) \\
o=\sqrt{2} \operatorname{tg} o=\frac{\mu_{1}-\mu_{2}}{\sqrt{\sigma_{1}{ }^{2}+\sigma_{2}^{2}}}
\end{gathered}
$$

Arec ces conventions :

$$
\left.\begin{array}{c}
m=\left(\mu_{1}+\mu_{2}\right) / 2 \\
\sigma=\sigma_{2}^{2} / 4\left(1+\lambda^{2}\right)\left(2+\delta^{2}\right) \\
\sqrt{\beta_{1}}=-3 \sin \varphi \cos ^{2} \varphi \cos 20 \\
1 / 2\left(\beta_{2}-3\right)=3 \cos ^{2} 2 \theta \cos ^{4} \varphi-2 \sin ^{4} \varphi
\end{array}\right\}
$$

Après élimination de 0 dans les deux dernières equations et après avoir posé $u=\sin ^{2}$, on a l'équation résolvante du système (2):

$$
6 u+3 u\left(\beta_{2}-3\right)-\beta_{1}=0
$$

Il est aisé de voir que, quelles que soient les distributions, cette équation possède toujours une racine et une seule comprise entre 0 et 1 . Les parametres sont donc entièrement déterminés par les équations (2) et (3).

Remarque. - Dans la troisième équation (2), mpparait la quantité $\sqrt{\beta_{1}}$. On désigne en général avec cette expression le rapport $u_{3} / \sigma^{3}$ ( $u_{0}:$ moment centré du troisième ordre), dont le carré est égal à $\beta_{1}$. Avec cette convention, on voit que $\sqrt{\beta_{1}}$ peut être négatif.

\section{3. - Critique de l'ajustement.}

Les débits journaliers ne constituant pas un chantillon de variables indépendantes issues d'une loi homogène, il n'est pas possible d'utilisor le test du $\chi^{2}$ pour juger de l'ajustement offectué sur l'ensemble des observations. Cette difficulté peut cependant être tournée en effecluant un tirage au hasard, avec remise, d'un tchantillon de taille donnée dans l'ensemble de lous les débits observés de la période étudiée.

Puisque la valeur de l'ajustement nous intéresse surtout pour les grands débits, il semble lont indiqué d'effectuer un tirage au hasard des $m$ plus grandes valeurs de $m$ échantillons de $n$ thits observés. La comparaison de cet échantillon avec la loi théorique de la plus srande de n. variables issues de la loi $\mathrm{F}$ dont la fonction de répartition est $G(x)=[F(x)]^{\prime}$, fournit alors un test d'ajustement pour les grands débits.

Le test du $\%^{2}$ est bien applicable ici. Il reste cependant une difficulté dans le choix du nombre de degrés de liberté intervenant dans la loi du $\%^{2}$. On sail, d'après la règle classique, que ce nombre y est égal à :

$$
v==k-p-1
$$

où :

$k$ est le nombre de classes construites avec l'échantillon:

$p$ et le nombre des parametres de la loi thérique estimés sur l'échantillon.

Or, les paramètres de la loi théorique sont ici estimés sur tous les débits et le test du $\%^{2}$ ne porte que sur une sous-population tirée au hasard de la population totale. En toute rigueur : v est done compris entre $k-5$ et $k-1$. Quoi qu'il en soit, on peut prendre $k$ assez grand compatible avec un échantillon assez petit par rapport au nombre total de débits de facon à ne commettre qu'une erreur négligeable en utilisant un $\chi^{2}$ avec $y=k-1$ degrés de liberté.

\section{4. - Applications.}

\section{a) PrLsentation des exmmples choisis.}

Ces applications ont porté sur 8 stations de jaugeages de caractères assez variés pour fournir un bon échantillonnage de divers régimes existant dans le pays.

Le tableau ci-après donne les valeurs des divers paramètres estimés à partir des observations. Ces valeurs ont été calculées en effectuant un découpage en classes du champ de variation des débits logarithmiques, ce qui a nécessité certaines corrections (corrections de Sheppard).

De ce tableau, on peut faire ressortir les valeurs de $\beta_{1}$ et $\beta_{2}$ du Rhin pour lequel les deux lois de Galton composantes sont pratiquement confondues.

Il faut aussi souligner les chiffres de la dernière colonne (coefficient de variation). Ce coefficient met en évidence la forte dispersion des débits des stations à régime purement pluvial (Blavet, Creuse) et de celles soumises aux influences méditerranćennes, exception faite pour le Verdon, dont la faible valeur de la dispersion est pour le moins étonnante.

\section{b) Calcul DU $\%^{2}$.}

Il nous reste à tester ces divers ajustements par la méthode décrite au paragraphe précédent.

Nous avons pris ici $m=100 . \quad n=10$. 


\begin{tabular}{|c|c|c|c|c|c|c|c|c|c|c|c|}
\hline STATIONS & $\begin{array}{l}\text { B. V. } \\
\mathrm{km}^{2}\end{array}$ & Période & $\dot{m}^{(1)}$ & $\sigma^{2(1)}$ & $\sqrt{\beta^{(1)}}$ & $\beta 2^{(1)}$ & $\mu_{t}$ & $\mu:$ & $\sigma_{t}$ & $\sigma_{2}$ & $\begin{array}{c}\text { Coefficient } \\
\text { de variation } \\
\\
\text { CV(a) }\end{array}$ \\
\hline Rhin (Rheinfelden). & 34.550 & $1939-54$ & 2,9578 & 0,0342 & 0 & 3 & 2,9578 & 2,9578 & 0,1849 & 0,1849 & 0,45 \\
\hline BLAVE' (Guerledan). & 620 & $1939-54$ & 0,7499 & 0,2126 & $-0,022$ & 2,273 & 1,1078 & 0,3920 & 0,2906 & 0,2906 & 1,44 \\
\hline Creuse (Eguzon)... & 2.400 & $1939-54$ & 1,0317 & 0.4262 & $-0,528$ & 3,208 & 0,6948 & 1,3685 & 0,6768 & 0,4090 & 2,92 \\
\hline TanN (Pinet) & 2.677 & $1939-54$ & 1,4903 & 0,1484 & $-0,484$ & 2,917 & 1,7272 & 1,2535 & 0,3621 & 0,2309 & 1,08 \\
\hline Truyère (Sarrans). & 2.462 & $1939-54$ & 1,2197 & 0,3422 & $-0,415$ & 2,820 & 0,8489 & 1,5906 & 0,5285 & 0,3606 & 2,26 \\
\hline $\begin{array}{l}\text { Gave DU Brousset } \\
\text { (Allias). }\end{array}$ & 61 & $1939-54$ & 0,3049 & 0.1586 & 0 & 2,108 & 0,6303 & 0,0205 & 0,2295 & 0,2295 & 1,15 \\
\hline $\begin{array}{l}\text { DORON DE BOZEL. } \\
\text { (La Perrière). }\end{array}$ & 301 & $1939-54$ & 0,9424 & 0,0592 & 0,201 & 1,760 & 1,1591 & 0,7257 & 0,1294 & 0,0884 & 0,01 \\
\hline VERDON (Quinson). & 1.661 & $1939-48$ & 1,3385 & 0,1081 & 0,361 & 2,337 & 1,5946 & 1,0825 & 0,2455 & 0,1609 & 0,88 \\
\hline
\end{tabular}

Les figures $I_{1}$ à $I_{S}$ montrent les histogrammes empiriques des 100 plus grandes valeurs de 100 échantillons de 10 débits tirés au hasard parmi les débits observés de la période étudiée. Les courbes continues figurent les lois théoriques déduites de l'équation : $\mathbf{G}(x)=[\mathbf{F}(x)]^{10}$.

Le test du $\%^{2}$ a donné les résultats suivants :

\begin{tabular}{|c|c|c|c|}
\hline COURS D'EAU & $\begin{array}{l}\text { Degrés } \\
\text { de liberté }\end{array}$ & $\% 0^{2}$ & $\begin{array}{c}\text { Prob. } \\
{\left[\%^{2} \geqslant \% \%^{2}\right]}\end{array}$ \\
\hline RHIN ... & 10 & 5,22 & 0,88 \\
\hline Blatvet ..... & 9 & 4,92 & 0,84 \\
\hline Creuse & 10 & 4,63 & 0,91 \\
\hline TARN . & 9 & 12,95 & 0,17 \\
\hline TRUYĖRE & 9 & 9,40 & 0,40 \\
\hline Gave du Brousset & 9 & 15,01 & 0,09 \\
\hline DORON DE BOZEL.. & 8 & 5,04 & 0,76 \\
\hline VERDON. & 9 & 13,76 & 0,13 \\
\hline
\end{tabular}

Pour la majorité des exemples traités, Ia probabilité de dépasser par hasard la valeur du $\%^{2}$ observée est assez forte. C'est donc l'indice d'un très bon ajustement. Seuls les $\%^{2}$ du Gave du Brousset et du Verdon soulèvent quelques difficultés du fait qu'ils sont proches du seuil de signification à $5 \%$. Après examen des graphiques $\mathrm{I}_{i,}$ et $\mathrm{I}_{\overline{7}}$, on peut cependant conclure que, si la distribution observée s'écarte significativement de la loi théorique, c'est dans le sens d'une plus petite dispersion des valeurs maxima.

En conclusion, la somme de deux lois de Galton s'adapte parfaitement bien aux courbes observées des débits classés. L'ajustement est particulièrement bor pour les grandes valeurs. Il est donc parfaitement légitime de substituer l'étude asymptotique de cette loi à l'étude de la courbe empirique, qui reste d'un maniement difficile pour les forts débits.

Dans la suite, cette loi ajustée sera désignée par la notation : loi $\mathbf{F}$.

Les distributions des valeurs extrêmes des échantillons de taille $n$ issus de cette loi seront désignées par : $\mathrm{F}_{n}$. 


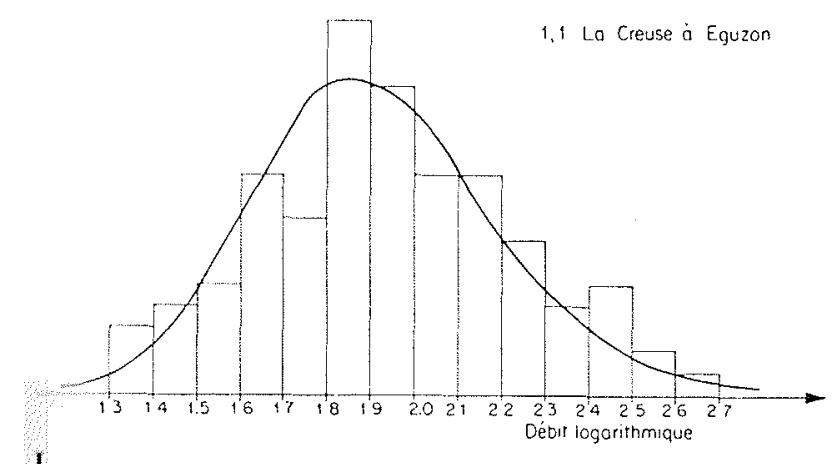

$I_{1}$

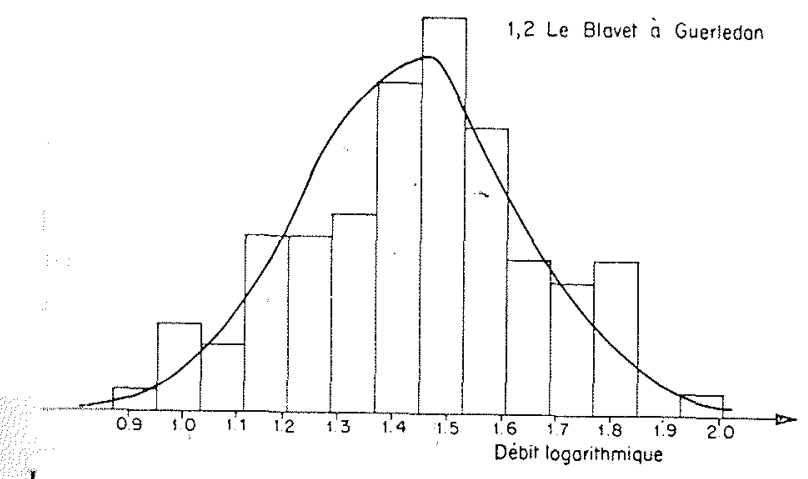

$1 \%$

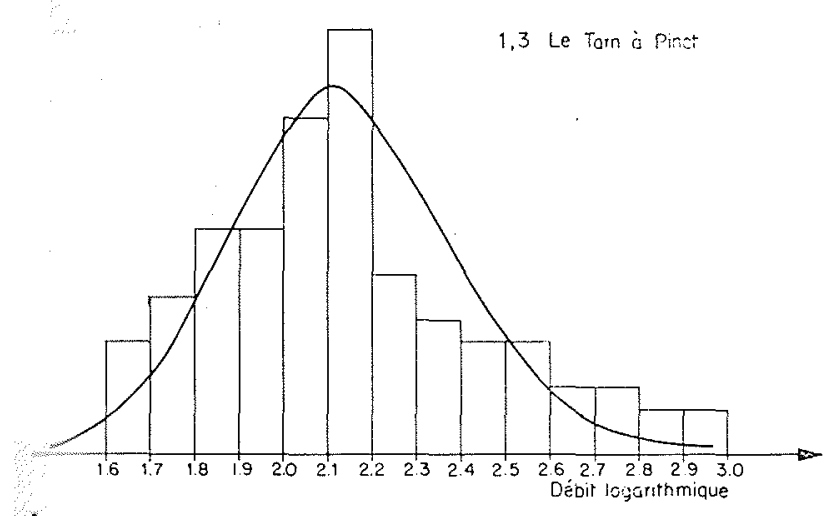

$I_{3}$

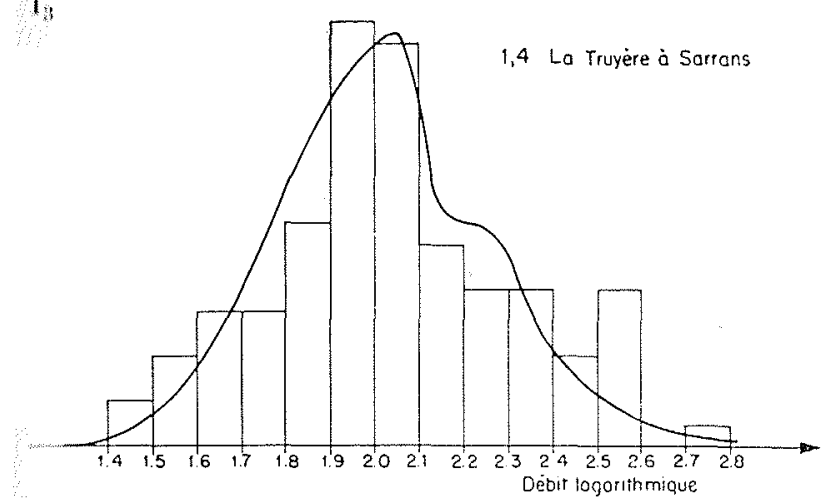

14

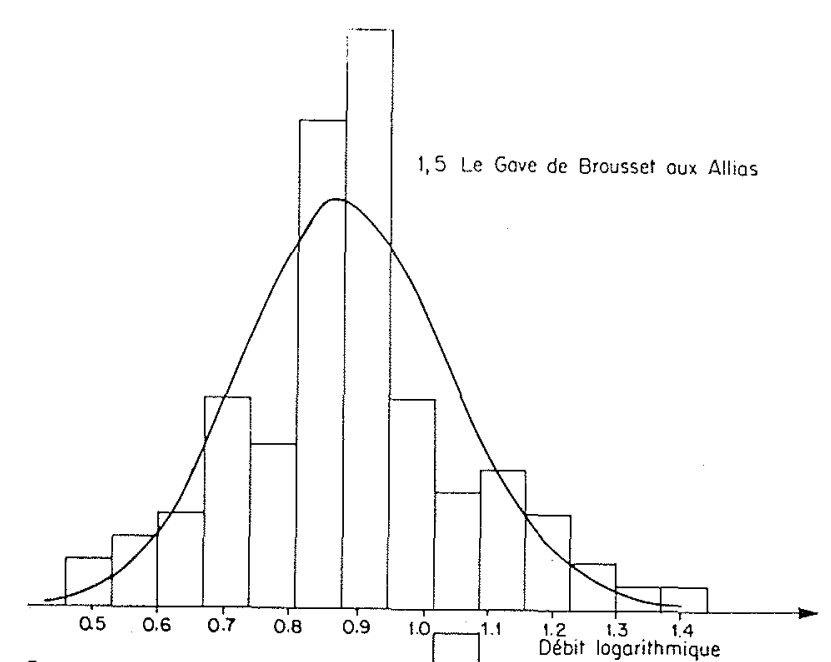

$\mathrm{I}_{5}$

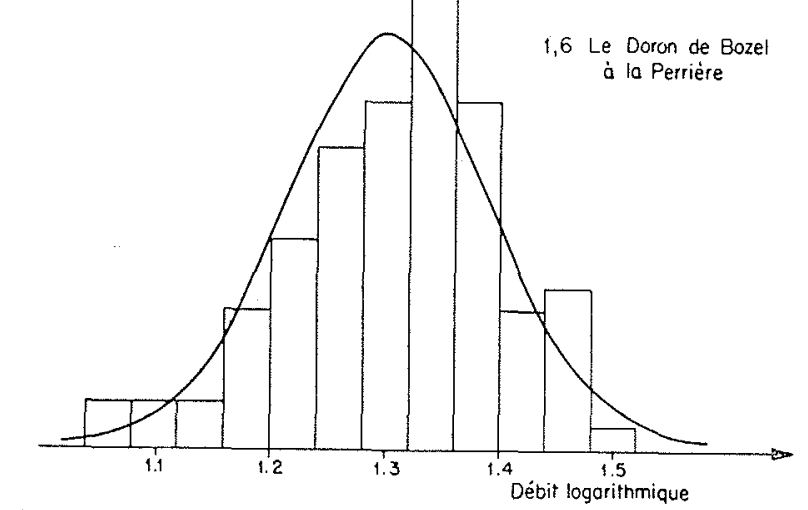

$I_{6}$

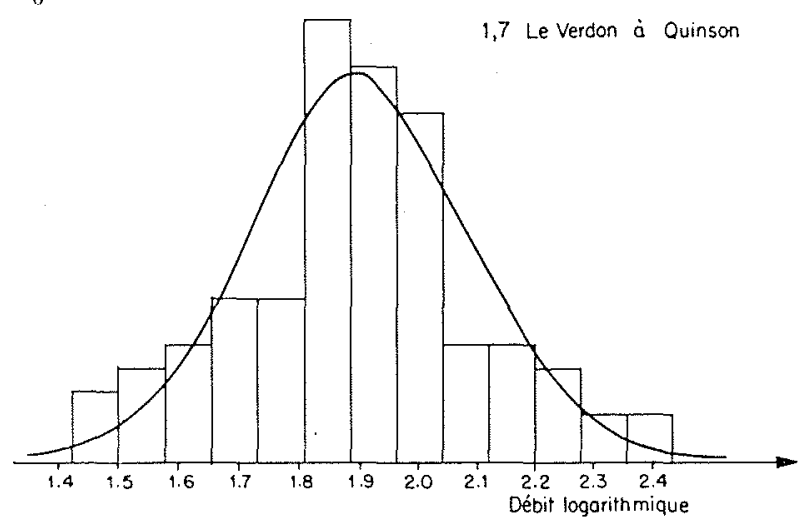

$\mathrm{I}_{\bar{\tau}}$

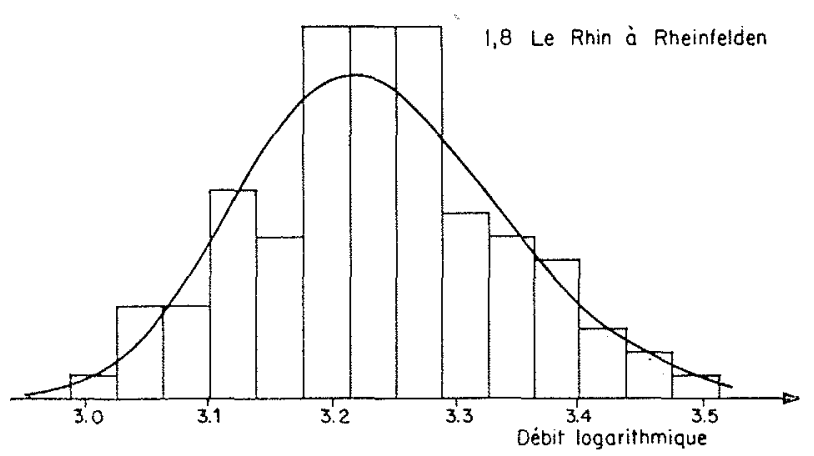

$\mathrm{I}_{\mathrm{S}}$

FIG. $I_{1} \wedge I_{B}$ 


\section{II. - COMPORTEMENTS ASYMPTOTIQUES COMPARES DES LOIS AJUSTÉES ET DE LA LOI DE GUMBEL}

La dernière étape de l'étude est la comparaison des comportements asymptoliques de la loi de Gumbel et de la loi des débits classés. De facon plus précise, il s'agit de savoir si, dans les limites les plus probables du champ de variation des débits de crue, la courbe des débits classés et la loi de Gumbel ont des comportements semblables.

Il est commode pour cela de prendre la loi de la plus grande de $n$ observations issues de la loi ajustée sur les débits classés et de la comparer à la loi de Gumbel.

Entendons-nous bien : dans le paragraphe précédent, nous avons pris des échantillons de grandes valeurs tirés de la distribution observée pour tester l'ajustement. Nous faisons maintenant des tirages à partir de la loi ajustée pour entrer dans le cadre de la théorie de Gumbel.

Nous prenons ici $n=100$. Ce chiffre n'a pas de valeur absolue. Il ne signifie pas qu'on fait l'hypothèse que la crue annuelle est la plus grande de 100 observations issues de la loi ajustée. On peut seulement dire que le champ de variation de cette loi $F_{100}$ recouvre à peu près, dans les cas les plus généraux, le champ de variation des débits de crue. Cela étant, les conclusions relatives à $F_{100}$ pourront alors être étendues à la loi des débits maxima annuels.

\section{1. - Loi de Gumbel ajustée et loi de Gumbel asymptotique.}

Ta loi de Gumbel s'exprime le plus généralement en fonction d'une variable réduite:

$$
\operatorname{Prob}[\mathrm{Y} \leqslant y]=\exp \left[-e^{-y}\right]
$$

variable réduite liée au débit $D$ par la relation :

$$
y=x(\mathrm{D}-u)
$$

Les parametres $\approx$ et $u$ sont asymptotiquement fonction de la loi initiale $\mathrm{F}$ :

$$
\mathrm{F}(\boldsymbol{l l})=\mathrm{I}-1 / n \quad \alpha=n f(u)
$$

Dans la deuxième formule:

$$
f(x)=d \mathrm{~F} / d x
$$

Ces relations fournissent une loi de Gumbel asymptotique qui est très éloignée dans le cas présent de la loi exacte $F_{10 n}$, comme le montrent les valeurs de $\alpha$ calculées par la formule précédente, comparées à celles obtenues en ajustant une loi de Gumbel aux débits de probabi-

\begin{tabular}{|c|c|c|}
\hline couns D'EAv & * (formule 4$)$ & $\alpha($ formule 5$)$ \\
\hline Rhin ... & 0,0059 & 0,0024 \\
\hline BLtVET . . . & 0,1644 & 0,0607 \\
\hline TruYÉRE & 0,0308 & 0,0101 \\
\hline
\end{tabular}
lité 0,5 et 0,9 de la loi exacte $F_{100}$.
Soit :

$$
\begin{aligned}
& 0,36651=\alpha_{100}\left(\mathrm{D}_{5} \cdots u\right) \\
& 2,25037=\alpha_{100}\left(\mathrm{D}_{9}, \cdots-u\right)
\end{aligned}
$$

\begin{tabular}{|c|c|c|}
\hline COURS D'WAY & $\alpha_{100}$ & $u_{100}$ \\
\hline Rhin . . . & 0,0024 & $2.438 \mathrm{~m} \quad \mathrm{~m}^{3} / \mathrm{s}$ \\
\hline BLAvET. & 0,0607 & $49,91 \mathrm{~m}^{3} / \mathrm{s}$ \\
\hline Creuse & 0,0071 & $183,3 \mathrm{~m}^{3} / \mathrm{s}$ \\
\hline TARN & 0,0077 & $287,0 \mathrm{~m}^{3} / \mathrm{s}$ \\
\hline TRUYĖRE . . . & 0,0101 & $215,7 \mathrm{~m}^{3} / \mathrm{s}$ \\
\hline Gave DU Brousset & 0,3305 & $12,56 \mathrm{~m}^{3} / \mathrm{s}$ \\
\hline DORON DE BOZEL. . & 0,3119 & $26,62 \mathrm{~m}^{3} / \mathrm{s}$ \\
\hline VERDON . . . . & 0,0311 & $123,3 \mathrm{~m}^{3} / \mathrm{s}$ \\
\hline
\end{tabular}

Il y a donc intérêt à utiliser la formule (5) de préférence à (4) pour déterminer la loi de Gumbel la plus apte à représenter correctement $\mathrm{F}_{100}$. Le tableau ci-dessous donne les valeurs de $\alpha$ et $u$ calculés par cette méthode :

\section{2. - Comparaison graphique.}

Pour comparer la loi $F_{100}$ el la loi de Gumbel ajustée, nous utilisons une methode graphique, qui a l'avantage sur les méthodes analytiques de présenter de manière plus simple el plus concrète les écarts entre les deux lois. Nous devons aussi nous attacher à prósontor des combes indépendantes des champs do vimitions différents des divers cours d'ean pour pouvoir effectuer des comparaisons valables 1.0 mophique II répond à cette exigence, 


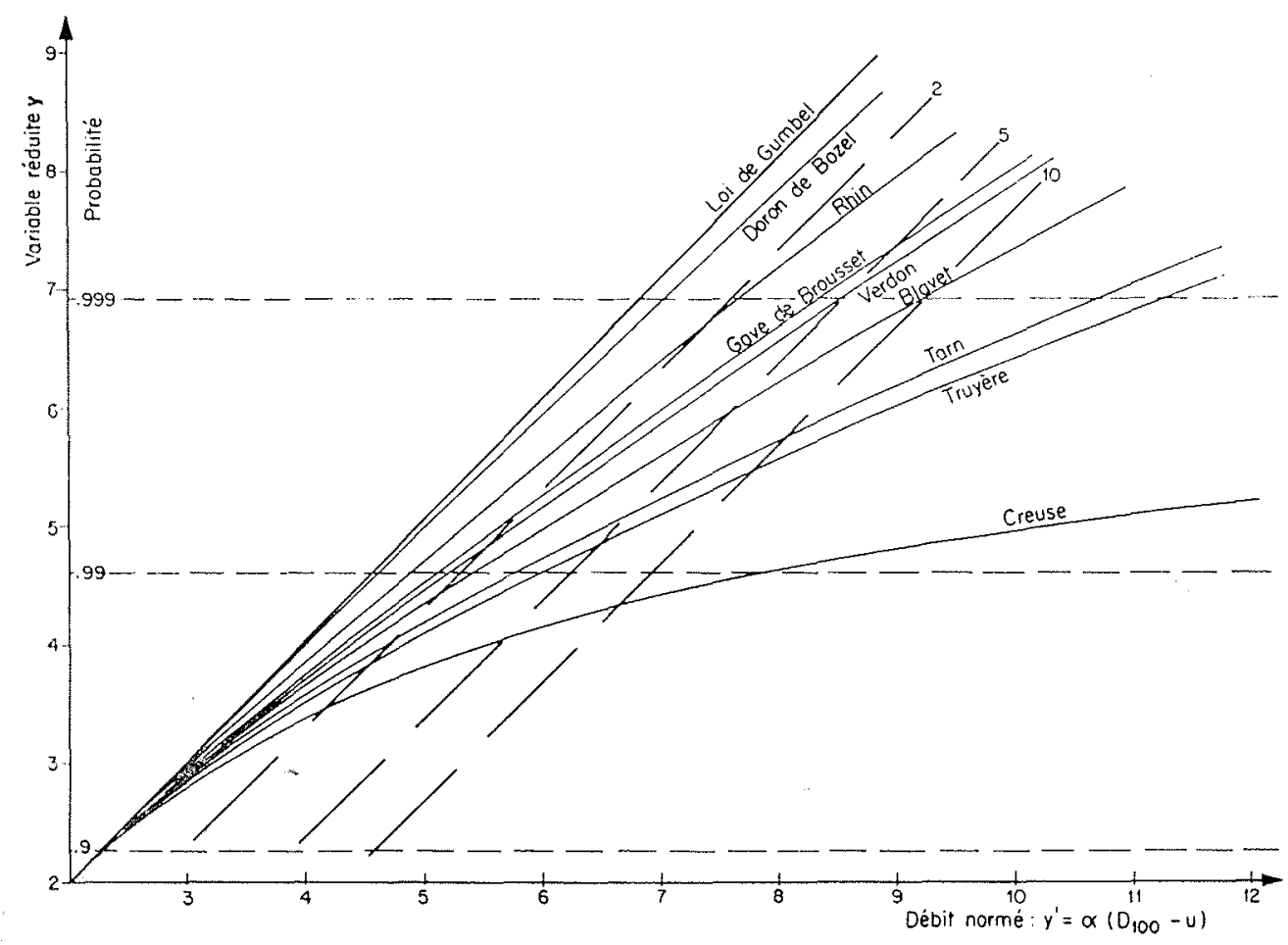

Fig. II

L.a figure présente :

4i In ordonnées, la variable réduite de Gumbel $y$ (l'échelle est aussi graduée en probabilités);

(1) In abscisses, la variable $y^{\prime}=\alpha_{100}\left(D_{100}-u_{100}\right)$ où $u_{100}$ et $\alpha_{100}$ sont déterminés de la façon décrite au paragraphe ci-dessus et $\vec{D}_{100}$ est le débit de la loi exacte $\mathbf{F}_{100}$ correspondant à une probabilité donnée.

Avec ces échelles, la loi de Gumbel exacte est rumósentée par la première bissectrice. Les segmunts de droite, parallèles à cette bissectrice, morqués 2, 5, 10, sont tels que les lois $F_{100}$ qui tes traversent présentent alors des périodes de revirence respectivement $2,5,10$ fois plus faiHes au moins que la loi de Gumbel ajustée. Ceci a pour intérêt d'indiquer de facon concrète les wrints entre les lois :

En se rapportant aux débits maxima annuels, (ii) peut, pour fixer les idées, admettre des wats allant jusqu'à 5 pour les valeurs milléunres (probabilité : 0,999), ceci, compte tenu des sircurs de mesure sur les grands débits. Si les twarts dépassent 5 et atteignent 10 et plus, ils toviennent inacceptables. (La crue centenaire devendrait millénaire avec la loi de Gumbel.)

\section{3. - Analyse du graphique II.}

En se basant sur les critères établis ci-dessus, on voit que la loi de Gumbel donne un ajustement :

-- excellent pour le Doron de Bozel,

- assez bon pour le Rhin;

- acceptable pour le Gave de Brousset et le Verdon;

- inacceptable pour le Blavet, le Tarn, la Truyère et la Creuse.

Pour ces quatre derniers cours d'eau, la loi de Fréchet, dont la décroissance asymptotique est plus lente, devra donner des ajustements meilleurs. Ces conclusions restent valables si le Verdon et le Gave du Brousset ont des lois moins dispersées.

Il est intéressant de confronter ces résultats avec les valeurs des coefficients de variation calculés plus haut. Il semble que la loi de Gumbel est d'autant moins valable que ces coefficients sont plus forts. On sait que cette forte dispersion est liée le plus souvent à l'influence pluviale que subit le cours d'eau. Là encore, le Verdon fait exception, qui présente un ajustement acceptable par la loi de Gumbel, bien que soumis fortement aux pluies méditerranéennes. 


\section{CONCLUSION}

Malgré les imperfections de la méthode, qui demande à être approfondie, les résultats de cette étude sont assez encourageants: ainsi les écarts des lois $\mathbf{F}_{100}$ à la loi de Gumbel semblent obéir à des règles précises en rapport avec le régime du cours d'eau. Cependant, le nombre des exemples traités est trop petit pour pouvoir lier de facon sûce le choix d'un type de loi (Fréchet ou Gumbel) aux caraclèes hydrologiques du bassin étudié. Pourlant la conclusion la plus claire qui se dégage de cette étude est que les influences pluviales doivent jotuer un rôle important surtout parce qu'elles sont la cause de la plus ou moins grande variabilité des débits.

\section{REFFÉRENCES}

[1] BLock (M.). - La distribution des débits d'un coups d'éa considéréc comme somme de deux distributions de Galton. La Métérologie, juillet 1939.

[2] Gimnat (R.). - Amenagement hydroélectrique des cours d'eau, statistique mathematique et calcul des probabilités. Revue Générale de l'Electricité, octobre 1932 .

[3] Gumber (E.-J.). - Méthodes graphiques pour l'analyse des débits de crue. La Honille Blanche, n" ${ }^{\circ}$ novembre 1956.

A la suite de cette communication à la Socićte Hydrotechnique de France, une discussion s'est engagée, dont nous donnons ci-après le compte rendu.

\section{DISCUSSION}

President : M. HUPNEn

Le Président remercie M. Brknier et pose quelques questions :

1. Pourquoi ne prend-on, pour comparer les deux lois, que la valeur médiane et celle de probabilité 0,9 , et non tous les éléments de l'échantillonnage?

M. Montat répond qu; ce choix, est en effet largement conventionnel. Il se justific ecpendant par ceci : lorsqu'on étudie une série de crues observées, on dispose au maximum de quelques dizaines d'observations, et les valeurs déciles et médianes ont un poids important dans l'ajustement. Il y a done intérêt à voir, ces valcurs stant fixées, comment les diverses lois divergent.

2" Peut-on conclure de cette comparaison qu'il n'y a pas de formule universelle et que les projeteurs d'ouvrages doivent appliquer l'une ou l'autre loi suivant le régime de la rivièe et le mécanisme de ses crues, et après avoir fait un certain nombre d'essais tendant a rechercher si une loi est plus convenable qu'une autre?

M. Berner pense qu'il en est bien ainsi et que l'on doit rechercher pour choisir entre les lois un critère qui ne soit pas soumis aux erleurs d'echantillonnage auxquelles sont sujettes les crues observees.

M. Mordat ajoute que, dans le cadre de la comparaison des lois de Gumbel et de Fréchet faite par M. Brrvier, on peut conclure que la plupart des courbes de debits classés peurent être ajustées avec ces deux seules lois, qui sont d'ailleurs de la mène famille, la loi de Fréchet pouvant être considéréc comme une généralisation de la loi de Gumbel. Cette conclusion limite done, en principe, le choix de l'utilisateur à ces deux seules lois.
3" Pourcuoi les lois du type Gallon sont-elles à rejeter, a priori pour l'étude asymptotique des débits classés.

M. Benxien répond que ces lois paraissent insuffisantes du fait qu'elles ne mettent en jeu que deux paramètres: on pourrat songer a utiliser ces lois en introduisant une valeur inférieure des débits, mais lestimation physique de cette valeur ne peut etre faite que graphiquement, e'est-âdire d'après une répartition empirique des débits observes, toujours soumise à un certain arbitraire qui devient, précisément, très important pour les grandes valeurs.

M. Charourher est frappé de la grande variéte des comportements asymptotiques des coubbes suivant les lois theoriques dont on est patli. La methode probabiliste apporte-t-elle plus que les methodes grossières appliquées jusqu'ici par les ingenienrs? Peut-on espérer que le calcul statistique serren un joll d'assez près la réalité pour qu'on puisse eviler los marges de sécurité souvent considérables que Pon ot obligo do garder, faute de mieux?

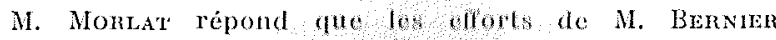
tendent précisément à fxor d thiblos déments connus, comme la courbe des dobits whrsts lat fonme de la loi qu'il faut ajuster; dans te condllons, si l'on peut analyser davantage los montrothoms physiques de la rivière et la chssen knwnit wh rogime, on voit, par

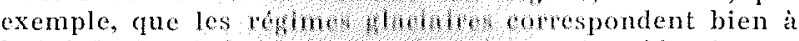

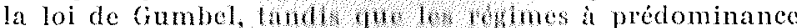
pluviale s'accommodoni wour 10 la lot de Fréchet. Ce 
Tistulat représente donc un certain progrès dans la veluetion de la marge d'incertitude que le calcul des pobabilités laisse encore aux ingénieurs et qui leur hipose sans doute une certaine prudence.

M. Chapouthen conclut que la physique doit s'assoFire aux calculs de probabilités pour les compléter.

M. le Président remercie M. Morlat et le félicite favoir bien tiré la philosophie de la discussion sur la vileur du calcul des probabilités.

Pour M. Gummer, l'incertitude des résultats du calcul thes probabilités en matière d'évaluation des débits Muxima de crues n'est pas imputable à une faille quelenique dans la structure mathématique des lois, mais 11 wae carence des données physiques : distributions iniwhes pratiquement inconnues, interdépendance d'un Whud nombre d'observations supposées indépendantes in cours d'une année et prises pour base, extrapolation to données portant sur un nombre assez faible d'années 200 a 30 ans) à des prévisions millénaires. Les mathémiliques ne sauraient suppléer ni à un manque de fwnse, ni à un manque d'observations. Cependant, il Nit legitime de supposer connues certaines propriétés mulytiques de ces distributions initiales, elles-même Imonnues, par exemple, la diminution de la densité de wohbilité des valeur's extrêmes suivant une loi expowenticlle qui suffira à établir la distribution asymptothuo de la plus grande valeur et définit la classe d'observillons nécessaires. Le nombre de ees observations Wepend du caractère de la distribution initiale; d'autro vinul l'aménágement hydro-électrique progressif des vrowes détruit ou réduit les possibilités de compa(ninon.

TYloal de l'ingénieur réside dans une seule observa1101 qui dome la réalité; mais statistiquement, une 01) ervation unique ne donne rien; il faut donc un grand rumbe d'observations, des études supplémentaires sur 16 enactere de la distribution initiale, un départ du munbe des observations indépendantes, toutes choses (wi) sont du domaine de la météorologie, non de la sta11. 14 gue.

Niwenant à l'exposé de M. Bennien, M. Gummer classe 1. Untributions des plus grandes valeurs en trois types: * punculielle (étudiée par M. Gumbel), distribution de C. wehy (étudiée par M. Fréchet), distribution à limite nimileure.

II "u lui semble pas nécessaire d'introduire une limul supérieure pour les crues; par ailleurs, l'introAmown d'une limite inférieure est sans intérèt pratique - Vithusalem ne meurt pas d'une maladie infantile $\gg)$. 11 wate done a comparer les deux premiers types de diswhution. Comme l'a dit M. Benvien, s'il y a un petit ammoro d'observations, et si le quotient de la plus W. nuic valeur à la plus petite n'est que de l'ordre de n11 3, ha distinction entre ces deux types est très Whilo, parce que l'allure des fonctions qui les repré*intent est pratiquement confondue dans un intervalle 4i: He reduit Dans ces conditions, il semble plus prudent 4 nulliger la loi de Fréchet qui aboutit à des extrapolaThu te débits plus grandes, quoique, du point de vue Thrue alle présente la difficulté que le débit moyen et f. Hurtype n'y existent pas. Pourtant on peut l'utiliser, fruma ru'elle corresponde à un meilleur ajustement, 6 Mil pir hlpothese seulement, doit donner une meilWhe extrapolation.

In donxième lieu, M. Gunber remarque que le travail 1. M. Broch, introduit dans l'exposé de M. Bennier, a 46 Tril a l'Université de Lyon, du temps où ce dernier y WInt ton éleve. M. Gumber estime que l'on pourrait mo ther aux facteurs $1 / 2$ et $1 / 2$ de $M$. Bloch, les poids I) $1 / \mathrm{B}$ en vue d'un meilleur ajustement.

1:ulli, la troisième partic du travail de M. Bernier consiste a traiter la distribution initiale d'une facon non asymptotique. M. Gunber demande quelle est la distribution exacte de la plus grande valeur à partir de la distribution initiale connue.

Il demande d'autre part à M. BErNier :

a) Pourquoi il emploie tantót $n=10$ et tantôt $n=100$;

b) Pourquoi, contrairement à toutes les autres, la courbe de la Truyère présente un point d'inflexion;

c) S'il at essayé de faire la comparaison avec une échelle linéaire, qui, selon M. Gumber, donne une meilleure représentation qu'en employant l'échelle logarithmique.

M. le Président remercie M. Gumbe de son exposé montrant les difficultés du problème; toutefois, parmi celles-ci, la détérioration des données initiales des rivières par suite des aménagements ne lui parait pas insurmontable, car les ingénicurs doivent pouvoil rétablir ces donnees et les reconstituer comme si la rivière etait dans son état naturel.

En ce qui concerne les poids B et 1-B proposés par M. Gumbel, M. Bernien estime que le fait d'utiliser des tacteurs multiplicatifs différents pour les deux lois de Galton composantes introduit un cinquième paramètre qu'il faut estimer a partir des observations. On court le risque d'avoir une loi ajustée trop souple. Mais il serait intéressant, à défaut de détermination statistique, de ehercher une détermination physique du rapport des deux lois composantes.

M. Bernisr répond, d'autre part aux questions $a$, $b$, et c de M. Gumber :

a) Les chiffres $n=10$ et $n=100$ de son exposé n'ont pas la mème signification.

Dans le premier cas, il s'agit de tester l'ajustement de la plus grande valeur de $n$ débits observés à la loi théorique : il a donc pris un $n$ assez petit (en l'occurrence 10) pour avoir un échantillon de grandes valeurs observées assez important.

Dans lel second cas, il s'agit d'avoir une représentation de la loi ajustée aux débits classés dans la zone du champ de variation des débits de crue. C'est ce qui impose un $n$ différent du premier chiffre utilisé et l'emploi de $n=100$.

b) Le point d'inflexion de la distribution relative à la Truyère tient à ce que la loi initiale de distribution est hétérogène (elle correspond ì deux lois de Galton superposées) la construction de ces courbes repose sur l'arbitraire du découpage en classes, de telle sorte que si les classes étaient plus petites pour les autres cours d'eau, on pourrait aussi distinguer des points d'inflexion dans les courbes qui représentent leur répartition.

c) Il n'a pas fait la comparaison avec l'échelle linéaire, mais il pense qu'il serait intéressant de voir l'allure des courbes à cette échelle.

M. Gumber fait alors, sur le sujet étudié, l'intervention suivante :

M. Bernier a employé la deuxième distribution asymptotique des plus grandes valeurs pour l'analyse des débits de crue. Mais il n'a pas utilisé la forme la plus générale de cette distribution.

Puisqu'une transformation linéaire ne change pas la forme d'une distribution statistique, on peut écrire la probabilité de Fréchet comme :

$$
F(x)=e^{-(u-\varepsilon / x-\varepsilon)^{r}}
$$

Cette distribution est de nouveau illimitée vers la droite. La signification de ll reste la même, mais, cette fois, il existe un troisième paramètre, à savoir 
la limite inferieure $\varepsilon$ des crues. Dans certains cas, l'introduction de e permettra un meilleur ajustement.

Malheureusement, l'estimation de a semble être difficile; $\varepsilon$ doit ètre positif; d'un autre côté, $\varepsilon$ doit être supérieur au plus petit débit de crue observé.

Pour le moment, je ne vois pas d'autres méthodes d'estimation de $\varepsilon$ que des approximations successives. On pose d'abord $\varepsilon=0$ et l'on obtient ainsi des estimations préliminaires $\widehat{\alpha}_{1}$ et $\widehat{u}_{1}$. Puis on doit choisir une nouvelle équation qui relie des valeurs observées on une observation $x$ proprement choisie a $\widehat{\alpha}_{1}, \hat{a}_{1}$ et $\hat{\varepsilon}$. Evidemment, le plus petit débit de crue sera roisin de la limite inférieure.

On obtiendra done une estimation de $\varepsilon$ en calculant la médiane $\breve{x}_{1}(\mathrm{~N})$ du plus petit débit de crue pour $\mathrm{N}$ années. Cette valeur est la solution : $x=\breve{x}_{1}(\mathrm{~N})$ de :

$$
1-e^{-(u-\varepsilon / x-\varepsilon) a}=e^{-\log 2 / N} \approx 1-(\log 2) N
$$

dont il résulte :

$$
(u-\varepsilon) /(x-\varepsilon))=[\log (1,44269 \mathrm{~N})]^{1 / \alpha}
$$

On remplacera $x=\breve{x}_{1}(N)$ par le plus petit débit de crue observé $x_{1}, u$ par $\widehat{u}_{1}$, a par $\hat{\alpha}_{1}$; alors l'estimation de $\varepsilon$ est :

$$
\frac{x_{1}[\log (1,44269 \mathrm{~N})]^{\widehat{1 / \alpha_{1}}}-u_{1}}{[\log (1,444629 \mathrm{~N})]^{1 / \widehat{a_{1}}-1}}
$$

A la limite, à savoir pour $\mathrm{N} \rightarrow \infty$, on obtient $\widehat{\varepsilon_{1}}=x_{1}$, quoique cette convergence soit assez lente. L'estimation de $\widehat{\varepsilon}$ est asymptotiquement «unbiased ». Elle peut être employée pourvu que $\widehat{\varepsilon}>0$, à savoir :

$$
\left(u / x_{1}\right) \alpha<\log (1,44269 \mathrm{~N})
$$

Celte condition peut etre vériliée immediatement d'après les observations.

Après l'estimation de $\hat{\varepsilon}_{1}$, on obtiendra une seconde estimation $\widehat{\alpha}_{22}$ et $\widehat{u_{2}}$ en traitant les 《observations》 $\log \left(x-\varepsilon_{1}\right)$ d'après la théorie linéaire employéc par M. Bernier (1).

L'introduction du troisième paramètre réduira la valeur de $g\left(x_{1}\right)$ à $\log \left(x_{1}-\varepsilon\right)$. L'influence diminue pour les grandes crues. Il en résulte que l'introduction de $\varepsilon$ n'améliorera pas l'accord entre la théorie et les observations si le plus petit débit de crue observé est inférieur à la valeur théorique obtenue par l'ajustement linéaire. C'est le cas dans les exemples traités par M. Bernier. Par contre, l'introduction de la limite inférieure des crues pourra améliorer l'accord entre la théorie et les observations, si le plus petit débit de crue est sensiblement supérieur à la valeur prévue par la théorie linéaire.

M. te Président remercie M. Benner ainsi que M. Gumnes, et donne la parole à M. Gunbel pour son deuxième exposé sur les débits d'étiage.

(1) (E.-J.) Gunnex. - Détermination commune des constantes dans les distributions des plus grandes valeurs, C.R.Ac.Sc., vol. 222, n" 1 , pp. 34-36, Paris, 1946.

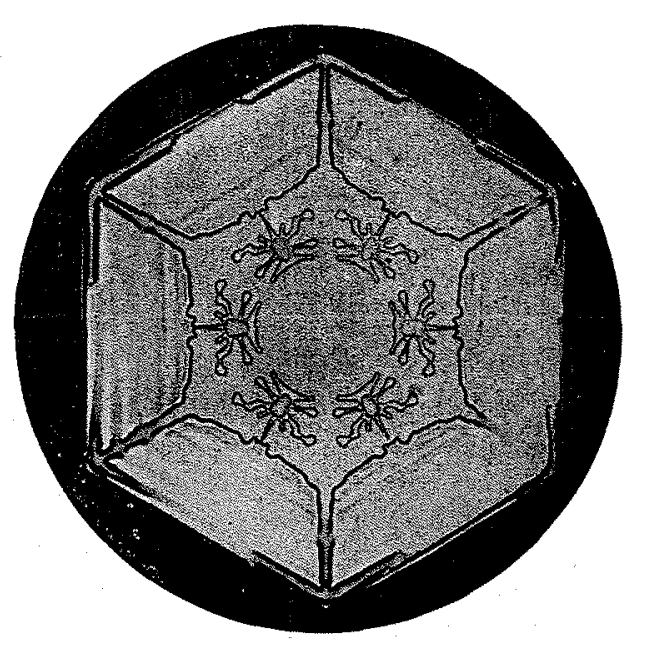

